\title{
SCIENTIFIC REPORTS

\section{Repeated Porphyromonas gingivalis W83 exposure leads to release pro-inflammatory cytokynes and angiotensin II in coronary artery endothelial cells}

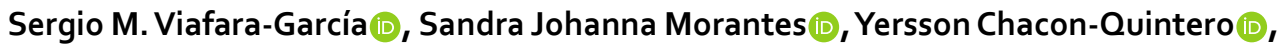

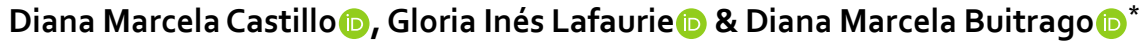

The role of Porphyromonas gingivalis ( $P$. gingivalis) or its virulence factors, including lipopolysaccharide (LPS) not only has been related with periodontitis but also with endothelial dysfunction, a key mechanism involved in the genesis of atherosclerosis and hypertension that involving systemic inflammatory markers as angiotensin II (Ang II) and cytokines. This study compares the effect of repeated and unique exposures of $P$. gingivalis W83 LPS and live bacteria on the production and expression of inflammatory mediators and vasoconstrictor molecules with Ang II. Human coronary artery endothelial cells (HCAEC) were stimulated with purified LPS of $P$. gingivalis $(1.0,3.5$ or $7.0 \mu \mathrm{g} / \mathrm{mL})$ or serial dilutions of live bacteria (MOI 1: $100-1: 0,1)$ at a single or repeated exposure for a time of $24 \mathrm{~h}$. mRNA expression levels of AGTR1, AGTR2, IL-8, IL-1 $\beta$ and MCP-1 were determined by RT-qPCR, and IL-6, MCP-1, IL-8, IL-1 $\beta$ and GM-CSF levels were measured by flow cytometry, ELISA determined Ang II levels. Live bacteria in a single dose increased mRNA levels of AGTR1, and repeated doses increased mRNA levels of IL-8 and IL-1 $\beta(p<0.05)$. Repeated exposure of live- $P$. gingivalis induced significant production IL-6, MCP-1 and GM-CSF ( $<$ < 0.05). Moreover, these MCP-1, IL-6 and GM-CSF levels were greater than in cells treated with single exposure $(p<0.05)$, The expression of AGTR1 and production of Ang II induced by live-P. gingivalis W83 showed a vasomotor effect of whole bacteria in HCAEC more than LPS. In conclusion, the findings of this study suggest that repeated exposure of $P$. gingivalis in HCAEC induces the activation of proinflammatory and vasoconstrictor molecules that lead to endothelial dysfunction being a key mechanism of the onset and progression of arterial hypertension and atherosclerosis.

Inflammation has received much attention as an determining factor in hypertension and atherosclerosis progres$\operatorname{sion}^{1,2}$. Although the effect of inflammation on endothelial dysfunction has been widely studied, the mechanisms of inflammation in hypertension have not been completely elucidated ${ }^{2}$.

Chronic systemic inflammation induced by periodontitis is linked to endothelial dysfunction due to the entry of Gram-negative anaerobes into the bloodstream after various stimuli, such as tooth brushing and chewing ${ }^{3}$ and periodontal treatment ${ }^{3-5}$ likewise, $\mathrm{DNA}^{6,7}$ of periodontal pathogens (e.g., Porphyromonas gingivalis) and live bacteria $^{8,9}$ have been demonstrated in atherosclerotic coronary lesions ${ }^{10,11}$, and increased systemic inflammatory markers induced by endotoxemia have been associated with hypertension/early atherosclerosis and periodontal disease ${ }^{12,13}$. Hence, there is a potential association between vascular inflammation and cardiovascular disease (CVD), since endothelial cells are the primary targets of immunological attack in inflammatory responses ${ }^{14}$.

The endothelium has been described as a secretory unit of pathogen-associated molecular patterns (PAMPs) that generates secreting soluble mediators. Cytokines and chemokines, as well as other products such as reactive oxygen species and metalloproteinases (MMPs) have been correlated with greater atheromatous lipid core 

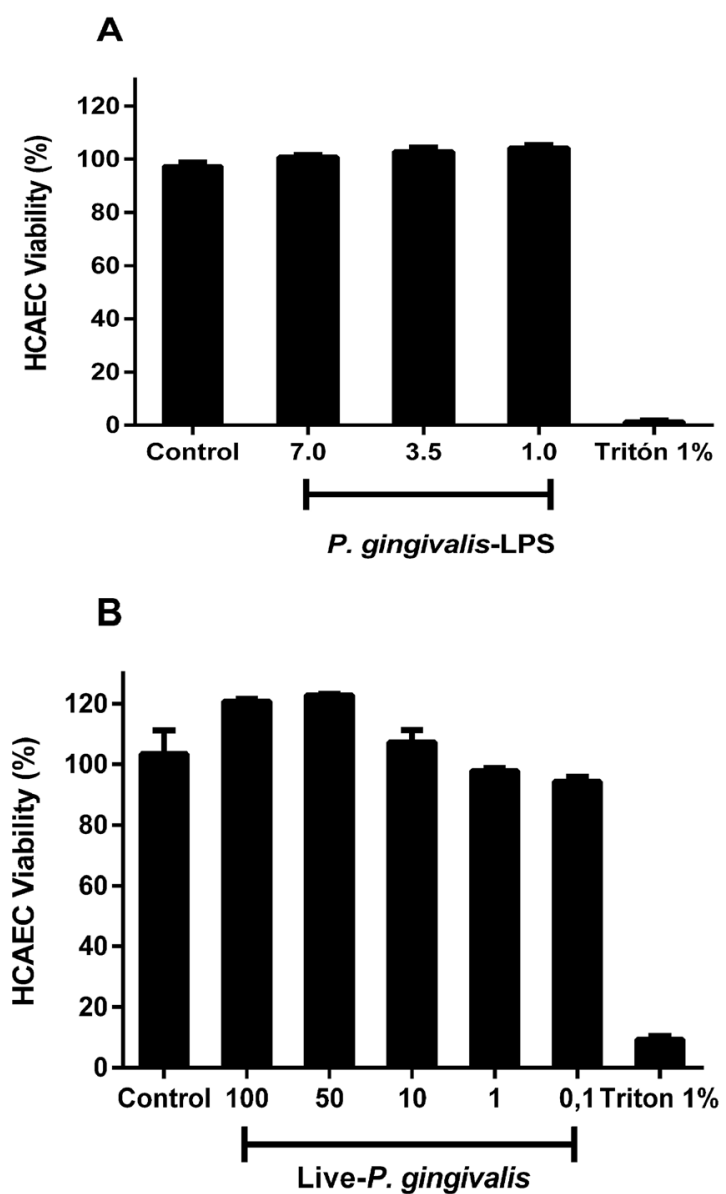

Figure 1. Viability of HCAECs after repeated treatments with live-P. gingivalis (A) and P. gingivalis-LPS (B). The HCAECs were stimulated to repeated live-P. gingivalis (MOI 1:100 - 1:0,1) and P. gingivalis-LPS (1.0, 3.5 and $7.0 \mu \mathrm{g} / \mathrm{mL}$ ) exposures, during $24 \mathrm{~h}$. Cell viability was determined according to the fluorometric detection after reduction of resazurin in the resorufin product using AlamarBlue. $1 \%$ was considered our positive control of cell death. Percentage of cell viability with respect to the control. *Represents the statistical difference with respect to the control or without stimulus. $(\mathrm{p}<0.05)$. Three independent experiments were performed; the results are presented as the means $\pm \operatorname{SEM}(\mathrm{n}=3)$.

increases in the presence of monocytes-macrophages and in the levels of IL-8, MCP-1, MMP-8 and MMP-9 in atherosclerotic plaques ${ }^{15,16}$. P. gingivalis, a major periodontal pathogen in periodontitis, has been shown to stimulate cytokine/chemokine production, which induces expression of cell adhesion molecules, including intercellular adhesion molecule (ICAM-1), vascular cell adhesion molecule (VCAM-1) and p-selectin, which are considered key steps in the onset of endothelial dysfunction ${ }^{17,18}$. P. gingivalis also activates endothelial cells, triggers smooth muscle cell proliferation and therefore impairs vasomotor function ${ }^{19,20}$.

The evolving role of Ang II as a regulator of endothelial cell function and its action by stimulating various receptors, specifically angiotensin II type 1 receptor (AGTR1) stimulates oxidative stress, fibrosis, cell proliferation and a release of cytokines and chemokines which in turn mediates tissue inflammation ${ }^{21,22}$. Recently, the evidence has indicated that low-grade inflammation may be involved in the development of hypertension ${ }^{23}$. In fact, the initiation or progression of periodontitis might involve a local renin-angiotensin system (RAS) activation ${ }^{21,22}$, that could lead to an increase in blood pressure, a decrease in nitric oxide, inflammation and development of atherosclerosis and endothelial dysfunction ${ }^{22,24,25}$.

Several bacteraemia episodes occur continuously in patients with periodontitis and are even induced by daily dental-care activities (i.e., brushing, flossing and chewing) ${ }^{26,27}$. Repeated episodes are also generated during intensive periodontal treatments that could induce an inflammatory acute response in the endothelium ${ }^{28,29}$. Although many reports examining $P$. gingivalis have demonstrated vascular endothelium activation through the selective recruitment of leukocytes to inflammatory foci in HCAEC via Toll-like receptor 2 (TLR2) after a single exposure to endotoxin ${ }^{16,30,31}$, it has been not studied in an in-vitro approach using repeated exposure in endothelial models of inflammatory responses. Therefore, these in-vitro cell models do not represent or mimic the vascular pathophysiology in patients with periodontitis, since the endothelium is exposed to repeated and accumulative doses of transient endotoxemia that could represent changes in the response, compared to the single exposure models. The purpose of this study was to compare the inflammatory and vasoactive response of Ang II of HCAEC stimulated with single and repeated exposure of lipopolysaccharide (P. gingivalis-LPS) and live-P. gingivalis W83. 

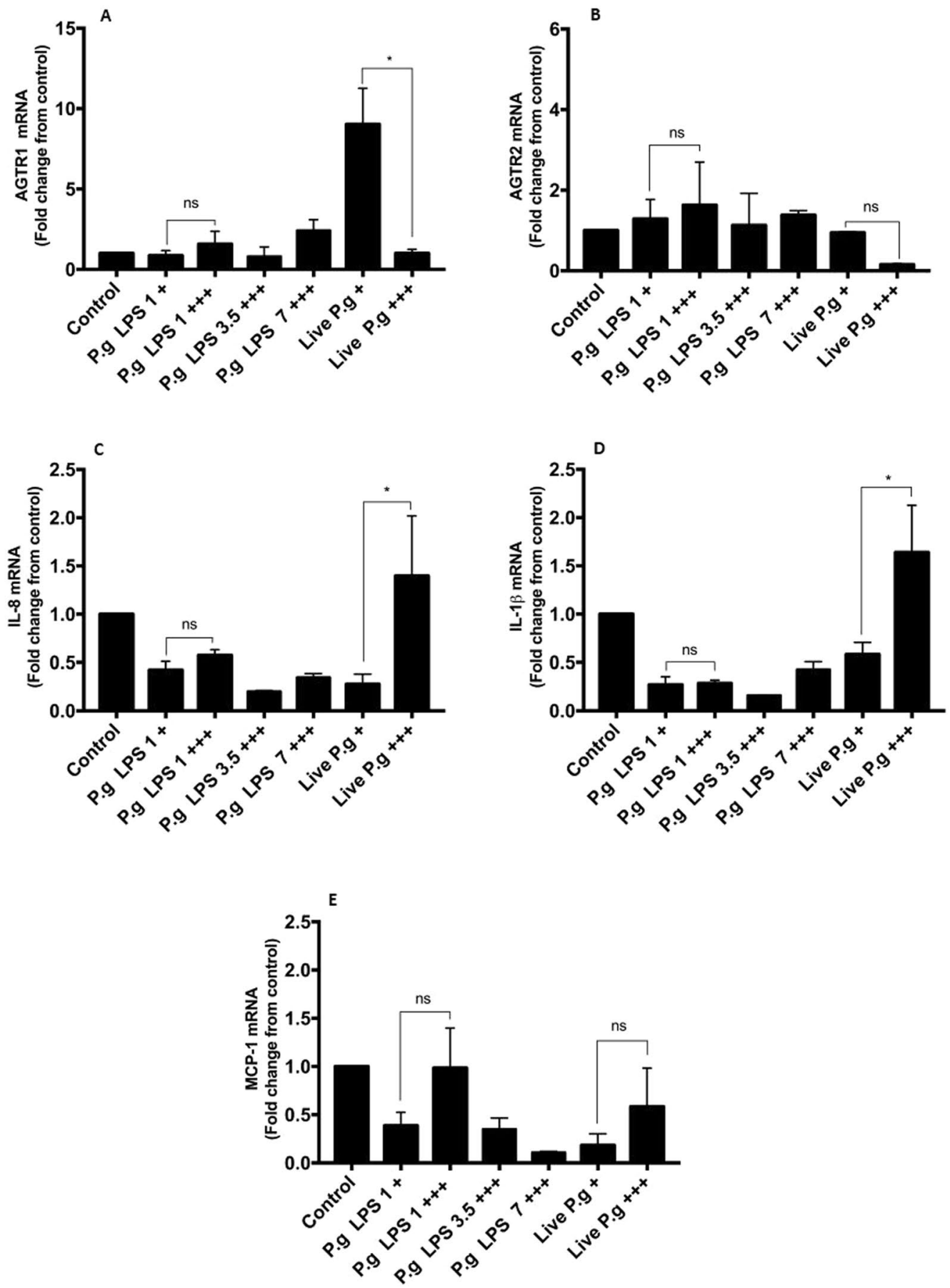

Figure 2. mRNA expression levels in HCAEC stimulated with $P$. gingivalis-LPS or live- $P$. gingivalis. Monolayers of HCAEC cultured in 12-well plates were stimulated with P. gingivalis-LPS $(1.0,3.5,7.0 \mu \mathrm{g} / \mathrm{mL})$ or serial dilutions of $P$. gingivalis (MOI 1:100- 1:0,1) for $24 \mathrm{~h}$ under repeated exposure $(+++)$ or single exposure $(+)$. After stimulation, (A) AGTR1, (B) AGTR2, (C) IL-8, (D) IL-1 $\beta$, (E) MCP-1, mRNA levels were measured by are expressed as the means by RT-qPCR. Results are expressed as the means \pm SEM $(n=3)$. Statiscal significance is represented as $* \mathrm{p}<0.05$, and (ns) for not significant.

\section{Results}

Effect of $\boldsymbol{P}$. gingivalis on the expression of AGTR1, AGTR2, IL-8, IL-1 $\beta$, MCP-1 in HCAEC. The biochemical characterization to confirm the degree of purity of LPS of $P$. gingivalis W83 showed a semi-rough LPS chemotype with bands of low molecular weight free of contaminants such as nucleic acids and proteins high endotoxic activity at very low concentrations similar to observed by commercial LPS (P. gingivalis ATCC 33277, InvivoGen) ${ }^{32}$. In relation to cell viability, our results demonstrated that LPS and live bacteria did not affect HCAECs (Fig. 1). 
In this study, we evaluated the expression of proinflammatory cytokine mRNA and AGTR genes in HCAEC stimulated with $P$. gingivalis-LPS and live-P. gingivalis $W 83$. The cells stimulated at single and repeated exposure with the different concentrations of $P$. gingivalis-LPS did not show significant changes for any of the pro-inflammatory markers even in comparison with the control group (Fig. 2), while repeated exposure of MOI 1:100 live- $P$. gingivalis compared to single exposure to the same MOI 1:100, significantly affects the expression of IL- 8 and IL-1 $\beta(\mathrm{p}<0.05)$. In contrast, the expression of MCP-1 was not significantly affected by the treatments; however, an apparent reduction in its mRNA was observed after single exposure to LPS and live bacteria. (Fig. 2A-E). On the other hand, a single exposure to live-P. gingivalis increased the AGTR1 expression (Fig. 2A) compared to unchallenged HCAEC and challenged with live bacteria to a repeated exposure $(\mathrm{p}<0.05)$, whereas AGTR2 (Fig. 2B) It was not because of the results of the treatments evaluated.

$P$. gingivalis induces IL-1 $\beta$, IL-8, IL-6, MCP-1, and GM-CSF production in HCAEC. In a pilot test, HCAEC cells were stimulated at day 1 , day 3 , and day 5 ; the supernatant was removed each $48 \mathrm{~h}$ to reaching a total of 7 days under repeated exposure. In general, we found similar results in a longer time frame and shorter time frame to induce the production of IL8 in the majority of LPS concentrations assessed (Supplementary Fig. 1). However, in a short period of time an overproduction of IL8 was achieved, suggesting greater endothelial activation than with a long period of time in which endothelial activation appears to decrease. This model could be used to study endotoxin tolerance of periodontal pathogens, an aspect that requires further research. Similarly, when comparing IL6 and IL1, there were also no modifications between the $24 \mathrm{~h}$ and 7 day in repeated exposure models (Supplementary Fig. 2).

The HCAECs were stimulated with $P$. gingivalis-LPS or live- $P$. gingivalis for $24 \mathrm{~h}$ by repeated or single exposures to evaluate the levels of IL- 8, MCP-1, IL-6, IL-1 $\beta$ and GM-CSF in culture supernatants. Repeated exposure to live- $P$. gingivalis induced a significant increase in the production of IL-6, MCP-1 and GM-CSF, compared to the control group $(\mathrm{p}<0.05)$, while the P. gingivalis-LPS is a single exposure only induced change in IL-1 $\beta$ (Fig. 3 ). The chemokine IL-8 was increased mainly in HCAEC challenged with live-P. gingivalis and $P$. gingivalis-LPS at $7 \mu \mathrm{g} / \mathrm{mL}$ at repeated expositions, but not significantly compared to the control (Fig. 3A). After considering the results so far, endothelial responses differed between approaches. HCAEC to repeated exhibitions with live-P. gingivalis achieved a significantly higher production of MCP-1, IL-6 and GM-CSF than treated endothelial cells at a single exposure of bacteria (Fig. 3B-D), while producing IL-1 $\beta$ (Fig. 3E) was higher in HCAEC challenged with LPS at a single exposure of $1 \mu \mathrm{g} / \mathrm{mL}(\mathrm{p}<0.05)$.

$P$. gingivalis increases angiotensin II levels in HCAEC. Ang II concentration was evaluated in HCAEC supernatants after $P$. gingivalis-LPS stimulation and live- $P$. gingivalis in single and repeated expositions. Only live-P. gingivalis (MOI 1:100) at repeated exposure significantly increased the concentration of Ang II, compared to the live bacteria single exposure (1.9 times) and the control cells (2.1 times) (Fig. 4).

\section{Discussion}

Oral pathogens associated with periodontitis, such as P. gingivalis, have been of particular interest due to the high levels of bacteraemia and endotoxemia after routine dental procedures and everyday oral activities, such as tooth brushing ${ }^{7}$. The biological mechanisms underlying the potential link between periodontitis and atherosclerosis or hypertension remain unclear, mainly in terms of inflammatory and vasoactive endothelial responses to $P$. gingivalis with repeated exposures. In this study, we evaluated the effects of single versus repeated administration of P. gingivalis-LPS and whole bacteria on the pro-inflammatory mediators and vasoactive markers involved in HCAEC atherosclerotic response.

In relation to cell viability, there are no reports in the literature that demonstrate the in vitro effect of periodontopathogens such as $P$. gingivalis on HCAEC cells at repeated exposures and at concentrations greater than $2 \mathrm{mg} / \mathrm{mL}$ with LPS ${ }^{33,34}$. Our results demonstrated that of $P$. gingivalis-LPS and the bacteria did not affect the cellular viability of HCAEC at the concentrations evaluated; data similar to those reported by Chou et al. ${ }^{35}$ where even $100 \mu \mathrm{g} / \mathrm{mL}$ of $P$. gingivalis-LPS W83 on leukocytes as PMN did not affect its viability ${ }^{36}$. However, depending on the type of cell line differences may occur, in this way have been described in esophageal cell lines as OE19 (adenocarcinoma), OE21 (squamous cell carcinoma), a sensitivity different from that reported in oral tumor cells (HN30), where the esophageal cancer cells were only sensitive to LPS P. gingivalis W83 concentrations of 20, 50 and $100 \mu \mathrm{g} / \mathrm{mL}(24,48$ and $72 \mathrm{~h}$ ), unlike oral cells (HN30) where the LPS increases the viability in relation to the control after $72 \mathrm{~h}$ of stimulus ${ }^{37}$.

P. gingivalis W83 strains have been known to adhere, invade and persist in bacteria-infected HCAEC ${ }^{33}$; however, its ability to activate endothelial cells by chemokine and cytokine production seems to exhibit slight activation to IL-8, IL-6, and MCP-1 compared to cells infected with other strains, such as 381 or $33277^{33}$. On the other hand, this study demonstrated inflammatory effect of W83 strain on endothelial cells, since HCAEC exposed to repeated exposure of live- $P$. gingivalis induced significant increases in chemokine levels, such as IL- 8 and MCP1 , and in cytokines, such as IL-6, compared with the control group, while HCAEC cells challenged with a single exposure of $P$. gingivalis W83 showed similar production of IL-8, IL-6 and MCP-1, compared with uninfected cells.

Increases in IL-8 are involved in the firm adhesion of rolling monocytes in the early stages of atherogenesis ${ }^{38}$. Similarly, IL-6 and MCP-1 increases have been implicated in the adhesion of leukocytes (mainly monocytes) to activated endothelium, which contributes to cellular migration ${ }^{39}$. Experimental findings suggest differential efficacy of $P$. gingivalis to activate HCAEC cells. P. gingivalis 381 also enhanced IL-6, IL- 8 and MCP-1 production and even the adhesion of immune cells with bacteria-infected HCAEC, while other P. gingivalis strains, such as W83, induced slight activation ${ }^{33,40,41}$. 

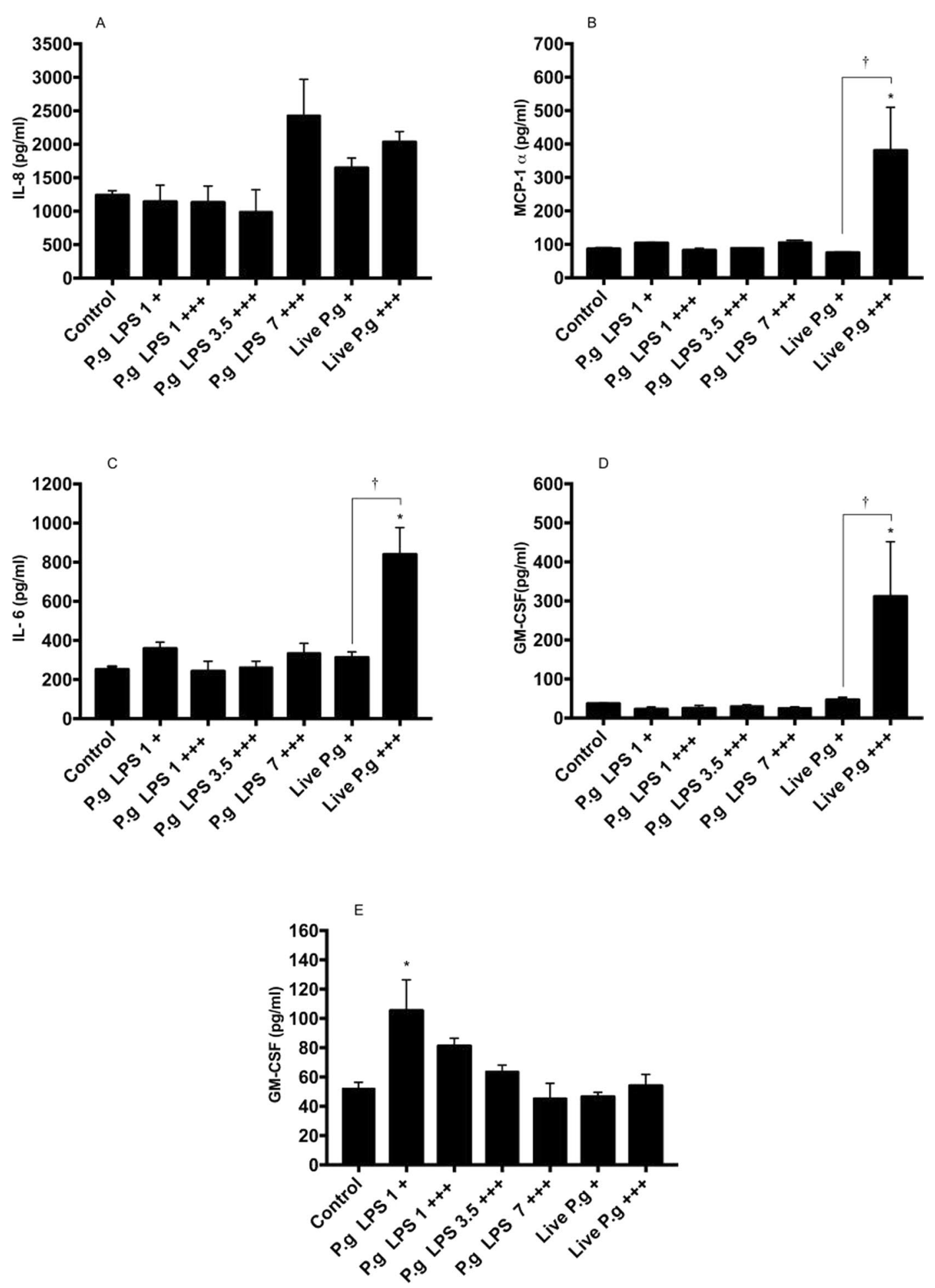

Figure 3. Chemokines and cytokines secreted in HCAEC stimulated with $P$. gingivalis-LPS or live-P. gingivalis. Monolayers of HCAEC cultured in 12-well plates were stimulated with P. gingivalis-LPS $(1.0,3.5,7.0 \mu \mathrm{g} / \mathrm{mL})$ or P. gingivalis (MOI 1:100 - 1:0,1) for $24 \mathrm{~h}$ under repeated exposure $(+++$ ) or single exposure $(+)$ ). After stimulation, levels of the following chemokines were measured in cell culture supernatants using a cytometric bead array: (A) IL-8, (B) MCP-1, (C) IL-6, (D) GM-CSF, (E) IL-1 $\beta$. Symbol (*) means p $<0.05$ vs control cells; (**) means $\mathrm{p}<0.01$ vs control cells; ${ }^{\dagger} \mathrm{p}<0.05$ compared vs cells treated by single exposure; ${ }^{\dagger \dagger} \mathrm{p}<0.01$ compared vs cells treated by single exposure; (ns) for not significant. Three independent experiments were performed; the results are presented as the means $\pm \operatorname{SEM}(\mathrm{n}=3)$. 


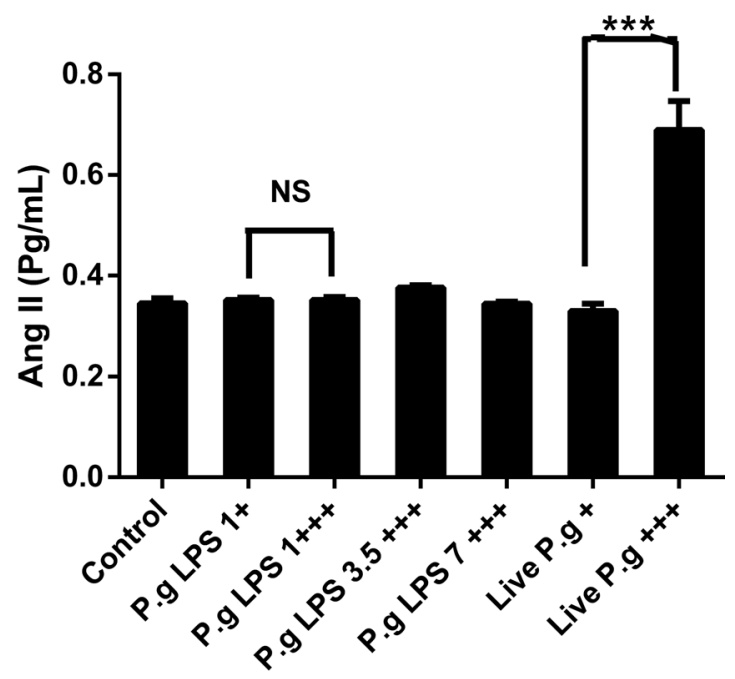

Figure 4. Angiotensin II levels are determined in the HCAEC cell culture supernatant stimulated to single $(+)$ or repetitive $(+++)$ exposures of $P$. gingivalis-LPS or live- $P$. gingivalis by the ELISA kit. The results are expressed as the means \pm SEM $(n=3)$ with a statistical significance represented as $(*) p<0.05$ vs. control, $\left(^{\dagger}\right) \mathrm{p}<0.05$ compared vs cells treated by single exposure; (ns) for not significant.

P. gingivalis-LPS was a poor inducer of IL-6, MCP-1, GM-CSF and IL-8 from HCAECs supernatants, similar results has been shown in immune cells as dendritic cell (DCs) ${ }^{42}$. In contrast, several authors have shown that endotoxin of $P$. gingivalis strain 33277 induces an important pro-inflammatory effect, increasing the production of IL-8, soluble E-selectin and MCP-1 in HCAEC ${ }^{43}$, while 381 strains only exerted a weak stimulatory effect on HCAEC $^{41}$. These results have been previously described and can be attributed to the presence of the K1 capsule or the characteristics of thechemotype of $P$. gingivalis-LPS W83 that may present differences in the O antigen region $^{44-46}$, which leads to the structural variations of the LPS that can be related with attenuation of the immune system of the host. Therefore, the low inflammatory response in HCAEC cells challenged with LPS could not be generalized to all strains of $P$. gingivalis ${ }^{16,33}$.

It has been described that $P$. gingivalis-LPS is an inducer of tolerance in macrophages, mainly by the suppression of the endothelial recognition receptor (TLR-4) $)^{47,48}$; however, our results showed that LPS $(7.0 \mu \mathrm{g} / \mathrm{mL})$ induced an increase in the release of IL- 8 at repeated exposures, which may be related to the type of endothelial recognition receptor to which $P$. gingivalis-LPS W83 binds (TLR-2) or other factors that may be involved by positively regulating the release of IL- $8^{49,50}$. However, new studies are required to elucidate the mechanism of action.

Regarding the relationship between mRNA and proteins levels, cytokines as MCP-1 and IL- 8 which were significantly detected in supernatants of HCAEC exposed to live-P. gingivalis, while MCP-1 mRNA levels were not upregulated. These discrepancies between mRNA and protein levels may involve the degradation rate of mRNA, which falls within a much tighter range ( $2-7 \mathrm{~h}$ for mammalian mRNAs vs $48 \mathrm{~h}$ for proteins $)^{51}$. In fact, a previous report in aortic smooth muscle cells has determined that in vitro half-life of MCP-1 mRNA is approximately $45 \mathrm{~min}^{52}$, however, further verification using transcriptome analysis or RT-qPCR are required.

On the other hand, Ang II has been implied in atherogenesis promoting the oxidative stress in the vasculature, endothelial dysfunction and induction of an inflammatory response in the vessel wall ${ }^{53}$. Regardless endothelial cells are not considered a dominant source of AngII and AGTR ${ }^{54}$; previous studies have shown that classic LPS from enterobacterias stimulate local and circulatory Ang II levels $s^{5-57}$. However, knowledge regarding the in vitro vasoactive effects of periodontopathogens as P. gingivalis-LPS on HCAECs, the cell type that has typically been used in studies of atherosclerotic diseases, is unclear yet. Contrariwise, our data showed P. gingivalis-LPS as a poor gene inducer to Ang II, AGTR1, AGTR2 and all chemokine/cytokine measured. It also suggests a weak vasoactive and inflammatory effect of endotoxin isolated from P. gingivalis W83.

Interestingly, repeated exposure of live bacteria $P$. gingivalis induces a greatest effect in HCAEC, suggesting that Ang II can modulate signaling cascades associated with the release of pro-inflammatory cytokines through calcium mobilization, arachidonic acid production, kinases activation (MAPKs, PKC, JAK, PI3K) or the activation of transcription factors as cAMP and NF-K $\beta^{17,58-60}$, which may explain the possible effects presented by the stimulation with live-P. gingivalis on endothelial cells ${ }^{21,55}$. However, further research is required to clarify possible mechanism.

Regarding the association between Ang II and the release of proinflammatory molecules such as IL8, IL6 and MCP1, there is no evidence with periodontopathogens, while some evidence have been reported Escheriria coli-LPS (E. coli-LPS) ${ }^{48,58,59}$, suggesting a synergistic effect between live-P. gingivalis stimulus and Ang II.

In contrast to the high concentration of Ang II at repeated dose of live bacteria, we found a down regulation of AGTR1 and AGTR2 at the mRNA level. Similar results with human saphenous vein cells (VSMC) and rat aortic smooth muscle (HASMC) suggest that high concentrations of Ang II can induce in vitro gen downregulation or desensitization/internationalization of AGTR $1^{48,58,59}$ or AGTR2 $2^{57}$. However, additional studies are required to identify the role of these receptors in endothelial dysfunction at repeated dose of periodontopathogens. 


\begin{tabular}{|c|c|c|}
\hline Gene & Primer Forward & Primer Reverse \\
\hline AGTR1 & 5'-TCAGCCAGCGTCAGTTTCAA-3' & 5'-GCCAGCAGCCAAATGATGATG-3' \\
\hline AGTR2 & 5'-GACAGACCAAACATATAAGAAGGA-3' & 5'-TCAGCTTGCTTAGTGCCTA-3' \\
\hline COX-2 & 5'-GATGATGTATGCCACAATCT-3' & 5'-AGTCTCTCCTATCAGTATTAGC-3' \\
\hline IL-8 & 5'-TGTGAAGGTGCAGTTTTGCCAAGG-3' & 5'-GTTGGCGCAGTGTGGTCCACTC-3' \\
\hline IL-1 $\beta$ & 5'-CTTTGAAGCTGATGGCCCTAAA-3' & 5'-AGTGGTGGTCGGAGATTCGT-3' \\
\hline MCP-1 & 5'-GAAAGTCTCTGCCGCCCTT-3' & $5^{\prime}$ - TTGATTGCATCTGGCTGAGCG-3' \\
\hline GAPDH & 5'-GGTGGTCTCCTCTGACTTCAACA-3' & 5'-GTTGCTGTAGCCAAATTCGTTGT-3' \\
\hline
\end{tabular}

Table 1. Primer sequences used for gene expression analysis by qPCR.

This work represents an alternative to the traditional in-vitro approach to evaluate $P$. gingivalis effects on endothelial cells, since transient and frequent bacteremia or endotoxemia episodes have been clearly described in patients with periodontitis. However, the classic stimulation model performed at a single dose up to $24 \mathrm{~h}$ did not expose the endothelium to these immunological challenges. Therefore, in-vitro exposure to more than one (repeated) exposure of $P$. gingivalis on the endothelium could lead to a better understanding for the study of endothelial dysfunction and pro-inflammatory activation.

\section{Materials and Methods}

Bacterial culture and inoculum standardization. P. gingivalis (BAA-308/W83) strain was obtained from the American Type Culture Collection (ATCC) and cultured using standard methods. This strain was originally isolated from humans with oral infections (i.e., periodontitis) and has been shown to be highly virulent compared with other $P$. gingivalis strains ${ }^{60}$. Bacteria were grown in supplemented Brucella agar $(0.3 \%$ Bacto agar, $0.2 \%$ yeast extract, $5 \%$ defibrinated sheep blood, $0.2 \%$ haemolyzed blood, $0.0005 \%$ hemin, and $0.00005 \%$ menadione) and incubated at $37^{\circ} \mathrm{C}$ for 4 days in anaerobic conditions (Anaerogen, Oxoid, Hampshire, UK) ${ }^{61}$. Bacterial inoculums were prepared and standardized for P. gingivalis in RPMI-1640 (Thermo Scientific, Waltham, MA, USA) and were quantified by spectrophotometry (Thermo Scientific, Waltham, MA, USA) at specific optical densities (OD) of $0.900-0.908$ at a wavelength of $620 \mathrm{~nm}$, corresponding to $2,6 \times 10^{9}$ bacteria/mL. The count of colony forming units (CFU) was confirmed in triplicate under incubation conditions. Viable bacteria experiments were performed in a maximum time of two hours after having counted them, in order to avoid bacterial mortality.

LPS extraction and purification. LPS extraction was performed using hot phenol-water, as previously reported $^{32}$, with $1.1 \mathrm{~g}$ of wetted $P$. gingivalis W83; the purification was accomplished using an enzymatic treatment with nucleases and protease, followed by size-exclusion chromatography (Sephacryl S-200 HR) with sodium deoxycholate as the mobile phase ${ }^{62}$. The characterization of the LPS was determined by SDS-PAGE electrophoresis, purpald assay and chromogenic LAL test, compared to the commercial LPS of P. gingivalis ATCC 33277 $(\text { InvivoGen })^{32}$.

Stimulation of HCAEC with LPS and viable bacteria in a single and repeated exposure model. HCAEC cells (LONZA, Walkersville USA) were cultured in supplemented EGM2 MV medium (LONZA, Walkersville, USA). The cells were used at passage 7 in growth medium $\left(2 \times 10^{5}\right.$ cells/well $)$ using 12 -well culture plates (CytoOne, USA Scientific, Orlando FL, USA) and pre-incubated at $37^{\circ} \mathrm{C}$ in a water-saturated atmosphere of $95 \%$ air and $5 \% \mathrm{CO}_{2}$ until reaching confluence at $20 \mathrm{~h}$. Subsequently, the cells were exposed to two treatment models at different concentrations of purified LPS $(1.0,3.5$ and $7.0 \mu \mathrm{g} / \mathrm{mL})$ and serial dilutions of live bacteria (MOI 1:100 - 1:0,1). A pilot study was conducted using longer exposure times where HCAEC cells were stimulated with P. gingivalis LPS on days 1, day 3 and day 5; the supernatant is removed every 48 hours to reach a total of 7 days under repeated exposure. The supernatant was stored at $-80 \mathrm{C}$, for subsequent cytokine analysis.

In the first model the cells were stimulated for $6 \mathrm{~h}$, after that, the stimulus was removed between each stimulus and replaced with a next exposure for another $6 \mathrm{~h}$ and for the last stimulation HCAEC the cells were exposed to another $12 \mathrm{~h}$, for a total exposure time from $24 \mathrm{~h}$. The supernatant was collected and storage for soluble factors measuring.

For the second treatment model, the conventional stimulus was referenced by literature in which HCAECs were stimulated with LPS at $1 \mu \mathrm{g} / \mathrm{mL}$ and serial dilutions of live bacteria MOI $(1: 100-1: 0,1)$ at a single exposure for $24 \mathrm{~h}^{43}$.

Cell viability assay. The cells were cultured in 96-well plates and stimulated according to the model described above with P. gingvalis-LPS $(1.0,3.5$ and $7.0 \mu \mathrm{g} / \mathrm{mL})$ and the live bacterium MOI 1:100. Subsequently to determine the cell viability was determined according to the fluorometric detection after the reduction of resazurin in the resorufin product using AlamarBlue (Biosource, Camarillo, CA, USA) ${ }^{63}$, the cells were placed in the medium containing $10 \%$ Alamar blue, after $10 \mathrm{~h}$ of incubation, $100 \mu \mathrm{L}$ of the medium was transferred to the wells of a 96-well plate and the changes in the fluorescence with a microplate fluorometer equipped with an excitation filter set of $560 \mathrm{~nm} / 590 \mathrm{~nm}$ emission (Infinite 200 PRO, Tecan, Männedorf, Switzerland). The unstimulated samples were considered our survival control, while those treated with $1 \%$ triton for 10 minutes were defined as positive control of death.

RNA extraction and RT-qPCR. The mRNA expression levels of AGTR1, AGTR2, IL-8, IL-1 $\beta$, MCP-1 and GAPDH were obtained from HCAEC cell stimulated to single or repeated exposures with LPS or live bacteria by qPCR. Total RNA was obtained from HCAEC using the QuickPrep MicroPrep isolation kit (Zymo Research, 
Irvine, CA, USA). The total amount of RNA was quantified using a Nanodrop (Thermo Scientific, Waltham, MA, USA). RT-qPCR was performed using $40 \mathrm{ng}$ of total RNA and a RT-qPCR Luna Universal One-Step kit (New England Biolabs, Ipswich, MA, USA). Primers were designed using Beacon Designer software and are listed in Table 1 . The reaction mixture consisted of $4,1 \mu \mathrm{L}$ of template $[10 \mathrm{ng} / \mu \mathrm{L}], 5 \mu \mathrm{L}$ of Luna Universal One-Step Reaction Mix [2x], 0,5 $\mu \mathrm{L}$ of Luna WarmStart RT Enzyme Mix [20x], and 0,2 $\mu \mathrm{L}$ of primers [10 $\mu \mathrm{M}]$, in a final reaction volume of $10 \mu \mathrm{L}$. The temperature profile used was as follows: $55^{\circ} \mathrm{C}$ for $10 \mathrm{~min}, 1 \mathrm{cycle}$ at $95^{\circ} \mathrm{C}$ for $1 \mathrm{~min}$, followed by 40 cycles of amplification at $95^{\circ} \mathrm{C}$ for $10 \mathrm{~s}$ and $60^{\circ} \mathrm{C}$ for $30 \mathrm{~min}$. Expression levels were calculated from the qPCR results based on the modified $2-\Delta \Delta \mathrm{Ct}$ method suggested by Pfaffl ${ }^{64}$. For these calculations, GAPDH and unstimulated cells were used as controls.

Determination of chemokine and cytokine levels by flow cytometry. IL-6, MCP-1, IL- 8 , IL-1 $\beta$, and GM-CSF levels were measured in culture supernatants from HCAEC stimulated with LPS or live bacteria by flow cytometry using a Human Proinflammatory Chemokine Panel (BioLegend, San Diego, CA, USA), according to the manufacturer's instructions. The minimal detectable concentrations of IL-8, MCP-1, IL-6, IL-1 $\beta$ and GM-CSF were 1.9, 2.2, 3.6, 2.6 and $2.0 \mathrm{pg} / \mathrm{mL}$, respectively. Flow cytometry was performed using the BD Accuri C6 flow cytometer (Becton Dickinson Biosciences, San Jose, CA, USA), and the data were processed using BD Accuri C6 Software.

Angiotensin II concentration. Ang II levels were determined from the supernatants of the cultured HCAEC stimulated with LPS or live bacteria, using the EIA ELISA Kit (Cayman Chemical, Ann Arbor, MI, USA). To detect Ang II in the supernatants, sample extraction and ELISA were performed according to the manufacturer's instructions ${ }^{65}$.

Statistical analysis. All experiments were repeated at least 3 times for qPCR and flow cytometry. All data were expressed as the mean \pm SEM. ELISA results were performed at least 3 times in duplicate. One-way variance analysis (ANOVA) and Tukey's post hoc tests were performed for all analyses. A p-value $<0.05$ was considered statistically significant.

\section{Conclusions}

Repeated exposure live-P. gingivalis induces a greater pro-inflammatory response than single exposure, described by IL-8, IL-6, MCP-1 and GM-CS in HCAEC. The expression of AGTR1 and production of Ang II induced by live-P. gingivalis W83 showed the vasomotor effect of whole bacteria in HCAEC more than LPS. The findings of this study suggest that repeated exposure of $P$. gingivalis in HCAEC induces the activation of proinflammatory and vasoconstrictor molecules that lead to endothelial dysfunction as a key mechanism of the onset and progression of arterial hypertension (HT) and atherosclerosis, which requires more research.

Received: 25 March 2019; Accepted: 8 October 2019;

Published online: 18 December 2019

\section{References}

1. Dinh, Q. N., Drummond, G. R., Sobey, C. G. \& Chrissobolis, S. Roles of Inflammation, Oxidative Stress, and Vascular Dysfunction in Hypertension. Biomed Res. Int., https://doi.org/10.1155/2014/406960 (2014).

2. Geovanini, G. R. \& Libby, P. Atherosclerosis and inflammation: overview and updates. Clin. Sci., https://doi.org/10.1042/cs20180306 (2018).

3. Forner, L., Larsen, T., Kilian, M. \& Holmstrup, P. Incidence of bacteremia after chewing, tooth brushing and scaling in individuals with periodontal inflammation. J. Clin. Periodontol., https://doi.org/10.1111/j.1600-051X.2006.00924.x (2006).

4. Horliana, A. C. R. T. et al. Dissemination of periodontal pathogens in the bloodstream after periodontal procedures: A systematic review. PLoS ONE, https://doi.org/10.1371/journal.pone.0098271 (2014).

5. Lafaurie, G. I. et al. Periodontopathic microorganisms in peripheric blood after scaling and root planing. J. Clin. Periodontol., https:// doi.org/10.1111/j.1600-051X.2007.01125.x (2007).

6. Kurihara, N. et al. Detection and localization of periodontopathic bacteria in abdominal aortic aneurysms. Eur. J. Vasc. Endovasc. Surg., https://doi.org/10.1016/j.ejvs.2004.08.010 (2004).

7. Figuero, E. et al. Detection of Periodontal Bacteria in Atheromatous Plaque by Nested Polymerase Chain Reaction. J. Periodontol., https://doi.org/10.1902/jop.2011.100719 (2011).

8. Kozarov, E. V., Dorn, B. R., Shelburne, C. E., Dunn, W. A. \& Progulske-Fox, A. Human Atherosclerotic Plaque Contains Viable Invasive Actinobacillus actinomycetemcomitans and Porphyromonas gingivalis. Arterioscler. Thromb. Vasc. Biol., https://doi.org/ 10.1161/01.atv.0000155018.67835.1a (2005).

9. Rafferty, B. et al. Impact of monocytic cells on recovery of uncultivable bacteria from atherosclerotic lesions. J. Intern. Med., https:// doi.org/10.1111/j.1365-2796.2011.02373.x (2011).

10. Pucar, A. et al. Correlation between atherosclerosis and periodontal putative pathogenic bacterial infections in coronary and internal mammary arteries. J. Periodontol., https://doi.org/10.1902/jop.2007.060062 (2007).

11. Gaetti-Jardim, E., Marcelino, S. L., Feitosa, A. C. R., Romito, G. A. \& Avila-Campos, M. J. Quantitative detection of periodontopathic bacteria in atherosclerotic plaques from coronary arteries. J. Med. Microbiol., https://doi.org/10.1099/jmm.0.013383-0 (2009).

12. Mustapha, I. Z., Debrey, S., Oladubu, M. \& Ugarte, R. Markers of Systemic Bacterial Exposure in Periodontal Disease and Cardiovascular Disease Risk: A Systematic Review and Meta-Analysis. J. Periodontol., https://doi.org/10.1902/jop.2007.070140 (2007).

13. Türkoğlu, O. et al. Evaluation of Systemic Levels of Neutrophilic Enzymes in Hypertensive Patients With Chronic Periodontitis. J. Periodontol., https://doi.org/10.1902/jop.2013.130346 (2014).

14. Harrison, D. G. et al. Inflammation, immunity, and hypertension. Hypertension, https://doi.org/10.1161/HYPERTENSIONAHA. $110.163576(2011)$.

15. Szekanecz, Z. \& Koch, A. E. Vascular endothelium and immune responses: Implications for inflammation and angiogenesis. Rheumatic Disease Clinics of North America, https://doi.org/10.1016/S0889-857X(03)00116-9 (2004).

16. Al-Soudi, A., Kaaij, M. H. \& Tas, S. W. Endothelial cells: From innocent bystanders to active participants in immune responses. Autoimmunity Reviews, https://doi.org/10.1016/j.autrev.2017.07.008 (2017). 
17. Andrukhov, O. et al. Different effects of Porphyromonas gingivalis lipopolysaccharide and TLR2 agonist Pam3CSK4 on the adhesion molecules expression in endothelial cells. Odontology, https://doi.org/10.1007/s10266-013-0146-x (2013).

18. Assinger, A. et al. Periodontopathogens induce soluble P-selectin release by endothelial cells and platelets. Thromb. Res., https://doi. org/10.1016/j.thromres.2010.10.023 (2011).

19. Wada, K. \& Kamisaki, Y. Roles of Oral Bacteria in Cardiovascular Diseases - From Molecular Mechanisms to Clinical Cases: Involvement of Porphyromonas gingivalis in the Development of Human Aortic Aneurysm. J. Pharmacol. Sci., https://doi.org/ 10.1254/jphs.09r22fm (2010).

20. Nakano, K. et al. Characterization of aortic aneurysms in cardiovascular disease patients harboring Porphyromonas gingivalis. Oral Dis., https://doi.org/10.1111/j.1601-0825.2010.01759.x (2011).

21. Santos, C. F. et al. Characterization of a local renin-angiotensin system in rat gingival tissue. J. Periodontol., https://doi.org/10.1902/ jop.2009.080264 (2009)

22. Santos, C. F. et al. Functional local renin-angiotensin system in human and rat periodontal tissue. PLoS One, https://doi.org/10.1371/ journal.pone.0134601 (2015).

23. Martin-Cabezas, R. et al. Association between periodontitis and arterial hypertension: A systematic review and meta-analysis. American Heart Journal, https://doi.org/10.1016/j.ahj.2016.07.018 (2016).

24. Gürkan, A. et al. Renin-angiotensin gene polymorphisms in relation to severe chronic periodontitis. J. Clin. Periodontol., https://doi. org/10.1111/j.1600-051X.2008.01379.x (2009).

25. Paizan, M. \& Vilela-Martin, J. Is There an Association between Periodontitis and Hypertension? Curr. Cardiol. Rev., https://doi.org $/ 10.2174 / 1573403 \times 10666140416094901$ (2014).

26. Geerts, S. O. et al. Systemic release of endotoxins induced by gentle mastication: association with periodontitis severity. J. Periodontol., https://doi.org/10.1902/jop.2002.73.1.73 (2002).

27. Tomás, I., Diz, P., Tobías, A., Scully, C. \& Donos, N. Periodontal health status and bacteraemia from daily oral activities: Systematic review/meta-analysis. Journal of Clinical Periodontology, https://doi.org/10.1111/j.1600-051X.2011.01784.x (2012).

28. D’Aiuto, F., Nibali, L., Mohamed-Ali, V., Vallance, P. \& Tonetti, M. S. Periodontal therapy: A novel non-drug-induced experimental model to study human inflammation. J. Periodontal Res., https://doi.org/10.1111/j.1600-0765.2004.00741.x (2004).

29. Tonetti, M. S., D’Aiuto, F. \& Nibali, L. Treatment of periodontitis and endothelial function. Japanese Journal of Chest Diseases, https:// doi.org/10.1056/NEJMoa063186 (2008).

30. Bainbridge, B. W. \& Darveau, R. P. Porphyromonas gingivalis lipopolysaccharide: An unusual pattern recognition receptor ligand for the innate host defense system. Acta Odontol. Scand., https://doi.org/10.1080/000163501750266710 (2001).

31. Hajishengallis, G. et al. Differential interactions of fimbriae and lipopolysaccharide from Porphyromonas gingivalis with the Tolllike receptor 2-centred pattern recognition apparatus. Cell. Microbiol., https://doi.org/10.1111/j.1462-5822.2006.00730.x (2006).

32. Gualtero Escobar, D. F. et al. Purificación y caracterización de lipopolisacáridos de Eikenella corrodens 23834 y Porphyromonas gingivalis W83. Rev. Colomb. Biotecnol., https://doi.org/10.15446/rev.colomb.biote.v16n1.44224 (2014).

33. Rodrigues, P. H. et al. Porphyromonas gingivalis Strain Specific Interactions with Human Coronary Artery Endothelial Cells: A Comparative Study. PLoS One, https://doi.org/10.1371/journal.pone.0052606 (2012).

34. Deng, H. et al. Invasion of four common periodontal pathogens into vascular endothelial cells in vitro. Zhonghua Kou Qiang Yi Xue Za Zhi (2010).

35. Chou, H. H. et al. Porphyromonas gingivalis fimbria-dependent activation of inflammatory genes in human aortic endothelial cells. Infect. Immun., https://doi.org/10.1128/IAI.73.9.5367-5378.2005 (2005).

36. Schaefer, J. S., Klein, J. R., Montufar-Solis, D., Jones, K. J. \& Ekhlassi, S. Differential Cytokine Patterns in Mouse Macrophages and Gingival Fibroblasts After Stimulation With Porphyromonas gingivalis or Escherichia coli Lipopolysaccharide. J. Periodontol., https://doi.org/10.1902/jop.2010.100226 (2010).

37. Gonçalves, M. et al. Effect of LPS on the viability and proliferation of human oral and esophageal cancer cell lines. Brazilian Arch. Biol. Technol., https://doi.org/10.1590/1678-4324-2016150485 (2016).

38. Zernecke, A., Shagdarsuren, E. \& Weber, C. Chemokines in atherosclerosis an update. Arteriosclerosis, Thrombosis, and Vascular Biology, https://doi.org/10.1161/ATVBAHA.107.161174 (2008).

39. Zohlnhöfer, D. et al. Adhesion of monocyte very late antigen-4 to endothelial vascular cell adhesion molecule-1 induces interleukin$1 \beta$-dependent expression of interleukin-6 in endothelial cells. Arterioscler. Thromb. Vasc. Biol., https://doi.org/10.1161/01. ATV.20.2.353 (2000).

40. Honda, T., Oda, T., Yoshie, H. \& Yamazaki, K. Effects of Porphyromonas gingivalis antigens and proinflammatory cytokines on human coronary artery endothelial cells. Oral Microbiol. Immunol., https://doi.org/10.1111/j.1399-302X.2004.00193.x (2005).

41. Roth, G. A. et al. Infection with a periodontal pathogen increases mononuclear cell adhesion to human aortic endothelial cells. Atherosclerosis, https://doi.org/10.1016/j.atherosclerosis.2006.03.018 (2007).

42. Pulendran, B. et al. Lipopolysaccharides from distinct pathogens induce different classes of immune responses in vivo. J. Immunol. (2001).

43. Nakamura, N. et al. Extended exposure of lipopolysaccharide fraction from Porphyromonas gingivalis facilitates mononuclear cell adhesion to vascular endothelium via Toll-like receptor-2 dependent mechanism. Atherosclerosis, https://doi.org/10.1016/j. atherosclerosis.2007.01.039 (2008).

44. Brunner, J. et al. The capsule of Porphyromonas gingivalis reduces the immune response of human gingival fibroblasts. $B M C$ Microbiol., https://doi.org/10.1186/1471-2180-10-5 (2010).

45. Rangarajan, M. et al. Identification of a second lipopolysaccharide in Porphyromonas gingivalis W50. J. Bacteriol., https://doi. org/10.1128/JB.01868-07 (2008)

46. How, K. Y., Song, K. P. \& Chan, K. G. Porphyromonas gingivalis: An overview of periodontopathic pathogen below the gum line. Frontiers in Microbiology, https://doi.org/10.3389/fmicb.2016.00053 (2016).

47. Verstrepen, L. et al. TLR-4, IL-1R and TNF-R signaling to NF- $\kappa$ B: Variations on a common theme. Cellular and Molecular Life Sciences. https://doi.org/10.1007/s00018-008-8064-8 (2008).

48. Outzen, E. M. et al. Lipopolysaccharides, but not Angiotensin 1l, Induces Direct Pro-lnflammatory Effects in Cultured Mouse Arteries and Human Endothelial and Vascular Smooth Muscle Cells. Basic Clin. Pharmacol. Toxicol., https://doi.org/10.1111/ bcpt.12697 (2017).

49. Zhang, T. et al. Aggregatibacter actinomycetemcomitans accelerates atherosclerosis with an increase in atherogenic factors in spontaneously hyperlipidemic mice. FEMS Immunol. Med. Microbiol., https://doi.org/10.1111/j.1574-695X.2010.00674.x (2010).

50. Kurita-Ochiai, T. \& Yamamoto, M. Periodontal Pathogens and Atherosclerosis: Implications of Inflammation and Oxidative Modification of LDL. Biomed Res. Int., https://doi.org/10.1155/2014/595981 (2014).

51. Vogel, C. \& Marcotte, E. M. Insights into the regulation of protein abundance from proteomic and transcriptomic analyses. Nat. Rev. Genet., https://doi.org/10.1038/nrg3185 (2012).

52. Liu, B., Poon, M. \& Taubman, M. B. PDGF-BB enhances monocyte chemoattractant protein-1 mRNA stability in smooth muscle cells by downregulating ribonuclease activity. J. Mol. Cell. Cardiol., https://doi.org/10.1016/j.yjmcc.2006.03.426 (2006).

53. Schmidt-Ott, K. M., Kagiyama, S. \& Phillips, M. I. The multiple actions of angiotensin II in atherosclerosis. Regulatory Peptides, https://doi.org/10.1016/S0167-0115(00)00178-6 (2000).

54. Matsusaka, T. et al. Liver Angiotensinogen Is the Primary Source of Renal Angiotensin II. J. Am. Soc. Nephrol., https://doi. org/10.1681/asn.2011121159 (2012). 
55. Ruiz-Ortega, M., Ruperez, M., Esteban, V. \& Egido, J. Molecular mechanisms of angiotensin II-induced vascular injury. Current Hypertension Reports, https://doi.org/10.1007/s11906-003-0014-0 (2003).

56. Sanchez-Lemus, E. et al. Angiotensin II at 1 receptor blockade decreases lipopolysaccharide-induced inflammation in the rat adrenal gland. Endocrinology, https://doi.org/10.1210/en.2008-0242 (2008).

57. Wang, F. et al. Angiotensin II type-1 receptor antagonist attenuates LPS-induced acute lung injury. Cytokine, https://doi. org/10.1016/j.cyto.2009.08.001 (2009).

58. Bkaily, G. et al. Angiotensin II AT 1 receptor internalization, translocation and de novo synthesis modulate cytosolic and nuclear calcium in human vascular smooth muscle cells. Can. J. Physiol. Pharmacol., https://doi.org/10.1139/y03-007 (2003).

59. Kranzhöfer, R. et al. Angiotensin induces inflammatory activation of human vascular smooth muscle cells. Arterioscler. Thromb. Vasc. Biol., https://doi.org/10.1161/01.ATV.19.7.1623 (1999).

60. Nelson, K. E. et al. Complete genome sequence of the oral pathogenic bacterium Porphyromonas gingivalis strain W83. J. Bacteriol., https://doi.org/10.1128/JB.185.18.5591-5601.2003 (2003).

61. Castillo, D. M. et al. Viability and effects on bacterial proteins by oral rinses with hypochlorous acid as active ingredient. Braz. Dent. J., https://doi.org/10.1590/0103-6440201300388 (2015).

62. Hirschfeld, M., Weis, J. H., Vogel, S. N., Ma, Y. \& Weis, J. J. Cutting Edge: Repurification of Lipopolysaccharide Eliminates Signaling Through Both Human and Murine Toll-Like Receptor 2. J. Immunol., https://doi.org/10.4049/jimmunol.165.2.618 (2014)

63. Oroszlán, M. et al. Proinflammatory changes in human umbilical cord vein endothelial cells can be induced neither by native nor by modified CRP. Int. Immunol., https://doi.org/10.1093/intimm/dxl023 (2006).

64. Pfaffl, M W. Institute of Physiology, FML-Weihenstephan, Center of Life and Food Sciences, Technical University ofMunich, G. A new mathematical model for relative quantification in real-time RT-PCR. Nucleic Acids Res., https://doi.org/10.1093/nar/29.9.e45 (2001).

65. Miceli, I. et al. Stretch reduces nephrin expression via an angiotensin II-AT 1 -dependent mechanism in human podocytes: effect of rosiglitazone. Am. J. Physiol. Physiol. https://doi.org/10.1152/ajprenal.90423.2008 (2009).

\section{Acknowledgements}

This study was supported by the Administrative Department of Science, Technology, and Innovation COLCIENCIAS (Grant: No. 130865740864) and by Universidad El Bosque, Research vice-rectory, Bogota, Colombia. The authors thank Juliette de Avila (Immunology laboratory, UIBO, El Bosque University, Bogota) for his assistance with flow cytometry and Oral Microbiology Laboratory, UIBO for their assistance in the culture of bacterium $P$. gingivalis.

\section{Author contributions}

D.M.B. and G.I.L. designed the original idea of the project; D.M.C. standardized the obtaining of the bacterial extract and the methodology of extraction and purification the LPS of P. gingivalis. S.V.G. and S.J.M. performed the mRNA and cytokine expression assays and the analysis of the results, Y.CH.Q., performed the assays for the quantification of Ang II, D.M.B. performed the cytotoxicity assays, D.M.B. directed the execution of the project and with Y.CH.Q. and G.I.L. they were in charge of the analysis of the results. All authors participated in the writing-original draft. S.V.G., D.M.B. and G.I.L. drafted the final manuscript, which was critically reviewed by all the authors.

\section{Competing interests}

The authors declare no competing interests.

\section{Additional information}

Supplementary information is available for this paper at https://doi.org/10.1038/s41598-019-54259-y.

Correspondence and requests for materials should be addressed to D.M.B.

Reprints and permissions information is available at www.nature.com/reprints.

Publisher's note Springer Nature remains neutral with regard to jurisdictional claims in published maps and institutional affiliations.

(c) (i) Open Access This article is licensed under a Creative Commons Attribution 4.0 International License, which permits use, sharing, adaptation, distribution and reproduction in any medium or format, as long as you give appropriate credit to the original author(s) and the source, provide a link to the Creative Commons license, and indicate if changes were made. The images or other third party material in this article are included in the article's Creative Commons license, unless indicated otherwise in a credit line to the material. If material is not included in the article's Creative Commons license and your intended use is not permitted by statutory regulation or exceeds the permitted use, you will need to obtain permission directly from the copyright holder. To view a copy of this license, visit http://creativecommons.org/licenses/by/4.0/.

(c) The Author(s) 2019 